\title{
The Characteristics Research of Solid-liquid Two-phase Fluid in the Filling Process of Fried Pepper Sauce
}

\author{
Qingni Yuan ${ }^{1 *}$, Qingyun Yuan ${ }^{2}$ and Feilong Du ${ }^{1}$ \\ ${ }^{1}$ Key Laboratory of Advanced Manufacturing Ttechnology (Guizhou University), \\ Ministry of Education, Guiyang 550003, China \\ ${ }^{2}$ Guiyang Dongsheng Patent and Trademark Affairs co., LTD, Guiyang 550002, China \\ E-mail: cme.qnyuan@gzu.edu.cn
}

\begin{abstract}
In order to improve stir uniformity in the filling process of fried pepper sauce, mixture of fried pepper sauce in stirred tank is as research object to study its flow characteristics. First, extreme theory of energy consumption is applied to analyze movement of solid-liquid two-phase flow in stirred tank. A mathematical model of movement relation of the two-phase flow is established based on the analysis. The computer then is used to numerical simulation analysis to obtain different sectional distribution of velocity field and density field for double helical structure. The results show as follows: the middle section of flow field in double helical structure has large average flow velocity, forms turbulence that has big intensity, wide range, and good liquidity. So it has good mixing effect, and it can realize stir uniformity in a wild range; but the flow characteristics significantly decrease in the corner of near wall field, and there are dead corner of stir. The calculation results can provide data basis for automatic filling technology.
\end{abstract}

Keywords: Fried pepper sauce, Solid-liquid two-phase, Numerical simulation, Velocity field.

\section{INTRODUCTION}

Fried pepper sauce is product that its raw material is mainly composed of dry red pepper particles and rapeseed oil. First, the raw material is fried in high temperature. After it is cooled, the product is then acquired by automatic filling process. During processing, pepper particles are immersed in rapeseed oil and absorb rapeseed oil, which cause pepper particles to sink in rapeseed oil and lead to non-uniform filling in the filling process. This is bottleneck problem in the processing of automatic filling [1].

The filling quality directly depends on mix uniformity of fried pepper sauce in stirred tank. The mixture in stirred tank mainly comprises pepper particles of solid phase and rapeseed oil of liquid phase, so it belongs to solid-liquid twophase fluid. In order to improve stir uniformity of mixture in stirred tank, the fluid characteristics of solid-liquid two-phase will be studied. In the paper, base on movement analysis of solid-liquid two-phase flow, the researches of fluid characteristics of two-phase include distribution of velocity field and density field in stirred tank of double helical structure, which will provide theoretical data basis for automatic filling technology.

\section{MOVEMENT ANALYSIS OF SOLID-LIQUID TWO- PHASE FLOW}

Fried pepper sauce is made by uniformly mixing pepper particles with rapeseed oil, so it is regarded as solid-liquid two-phase fluid. Its flow velocity distribution is a kind of mixed fluid velocity distribution that is connected with density and itself characteristics of pepper particle. At present, on the impact of suspended particles on flow velocity distributing, its functional relationship is establish between parameters to reflect distribution uniformity of the flow velocity and some factors such as fluid density, pepper particle size, or Richardson number to reflect density gradient, but they only obtain some obvious rule to some extent [2-5]. Flow velocity distribution of solid-liquid twophase is a multivariate compound function. It is a problem of turbulence flow that flow velocity distribution is not necessarily same direction with density change of suspended particles in a certain direction [6-8]. Therefore, extreme theory of energy consumption is used to describe it in order to find right combination relationship between these variables.

\section{1 Extreme theory of energy consumption}

For more phase fluid, when it flows under given initial and boundary conditions in a separate system, the density, velocity and pressure distribution are not only to satisfy 
continuity equation and energy equation, but also always automatic adjust at any time to make system energy consumption rate to be an extreme value. This is basic thought for extreme theory of energy consumption. When it is described by mathematical form under certain condition, it is an extreme problem of math $[9,10]$.

Mathematical extreme problem have extreme value problem of known function and functional extreme value problem of undetermined function. For functional extreme value problem of undetermined function, it may be expressed as integral form. When it is not expressed as integral form, calculus of variations is used to solve the extreme problem [11].

\section{2 Building mathematic model}

The process of fried pepper sauce in stirred tank is a complex flow of solid-liquid two-phase fluid [12, 13]. In order to realize its numerical simulation, there are some assumptions as follows:

1) Liquid phase (rapeseed oil) is incompressible fluid, and solid phase (pepper particle) is continuous medium. Physical properties of each phase are constant $[14,15]$.

2) Pepper particle is spherical, its size is uniform, and Phase transition is not considered [16].

According to extreme theory of energy consumption, if there are density distribution and velocity distribution of twophase, the actual energy consumption rate always reaches at an extreme value. In two-dimensional steady uniform fluid, when solid particles are fully suspended and balanced, mathematical expression of the theory is [10]

$\int_{0}^{H} \gamma_{m} u_{m} J d y=$ extreme value

where $\gamma_{m}=\left(r_{s}-\gamma\right) C+\gamma, \mathrm{H}$ is depth of rapeseed oil in stirred tank; $\mathbf{J}$ is gradient; $u_{m}$ is shorthand notation of $u_{m}(y)$, and flow velocity distribution of mixture; $\mathrm{C}$ is shorthand notation of $\mathrm{C}(\mathrm{y})$, and is vertical distribution of density; $r_{s}$ is volumetric number of particles; $r$ is volumetric number of liquid; $r_{m}$ is volumetric number of mixture. Equation (1) reflects extreme value with density distribution (C) and flow velocity distribution $\left(u_{m}\right)$.There are following constraints:

Density distribution (C) and flow velocity distribution $\left(u_{m}\right)$ satisfy continuous equation of suspended particles in the twophase flow:

$\omega C+K_{s} \frac{d C}{d y}=0$

and energy equation:

$r_{m} u_{m} J=\tau_{m} \frac{d u_{m}}{d y}-\frac{d\left(u_{m} \tau_{m}\right)}{d y}$

where $K_{s}=K^{2} y^{2} \frac{d u_{m}}{d y} ; \tau_{m}=K^{2} y^{2} \frac{\gamma_{m}}{g}\left(\frac{d u_{m}}{d y}\right)^{2}, \omega$ is particle falling velocity in rapeseed oil; $K_{s}$ is exchange coefficient of momentum; $\tau_{m}$ is shearing force of solid-liquid mixture; $\mathrm{K}$ is Carmen coefficient $K=0.4$. C and $u_{m}$ is obtained by
Equation (1) -(3), which uses variation method to solve. Equation (1) -(3) is converted by calculus of variation.

$$
\begin{aligned}
\Pi= & \int_{0}^{H} F\left(C, \frac{d C}{d y} ; u_{m}, \frac{d u_{m}}{d y}\right) d y \\
= & \int_{0}^{H}\left[(1+\beta) \frac{\gamma_{m}}{\gamma} u_{m} J-\beta \frac{\gamma_{m}}{\gamma} \frac{K^{2} y^{2}}{g}\left(\frac{d u_{m}}{d y}\right)^{2}\right. \\
& \left.+\lambda \omega C+\lambda K^{2} y^{2} \frac{d u_{m}}{d y} \bullet \frac{d C}{d y}\right] d y=\text { extreme value }
\end{aligned}
$$

where $\beta, \lambda$ is Lagrangian multiplier, and may be any constant. According to extreme condition of functional, the following equation is obtained:

$$
\begin{aligned}
\delta \Pi= & \int_{0}^{H}\left\{(1+\beta) \frac{\gamma_{\mathrm{s}}-\gamma}{\gamma} u_{m} J-\beta \frac{\gamma_{s}-\gamma}{\gamma} \frac{K^{2} y^{2}}{y}\left(\frac{d u_{m}}{d y}\right)^{3}\right. \\
& \left.+\lambda \omega-\frac{d}{d y}\left(\lambda K^{2} y^{2} \frac{d u_{m}}{d y}\right)\right\} \delta C d y \\
& +\int_{0}^{H}\left\{(1+\beta) \frac{\gamma_{m}}{\gamma} J+\frac{d}{d y}\left[3 \beta \frac{K^{2} y^{2}}{g}\left(\frac{d u_{m}}{d y}\right)^{2}-\lambda K^{2} y^{2} \frac{d C}{d y}\right]\right\} \delta u_{m} d y=0
\end{aligned}
$$

Boundary conditions are

$$
\left\{\begin{array}{c}
\left.u_{m}\right|_{y=H}=\left.u_{\max } \quad \frac{d u_{m}}{d y}\right|_{y=H}=2.5 \frac{u_{*}}{H} \\
C=\left.\right|_{y=H}=\left.0 \quad \frac{d C}{d y}\right|_{y=H}=0
\end{array}\right.
$$

The boundary condition (6) into equation (5) is

$$
\begin{aligned}
& u_{m} J-\frac{K^{2} y^{2}}{g}\left(\frac{d u_{m}}{d y}\right)^{3}+\left(\frac{0.01 K^{2} U^{3}}{g H \omega}-\frac{3 U J}{\omega}\right) \omega /\left\{\left(1-\frac{K u_{*}}{\omega}\right)\right. \\
& \left.-\frac{d}{d y}\left[K^{2} y^{2}\left(0.01 K^{2} \frac{U^{3}}{g H \omega}-\frac{3 U J}{\omega}\right) \bullet \frac{d u_{m}}{d y}\right]\right\}=0 \\
& 3 \frac{\gamma_{m}}{\gamma} J+\frac{d}{d y}\left[3 \frac{\gamma_{m}}{\gamma}\left(\frac{d u_{m}}{d y}\right)^{2} \frac{K^{2} y^{2}}{g}\right. \\
& \left.-\frac{\left(\gamma_{s}-\gamma\right) K^{2} y^{2}}{\gamma\left(1-\frac{K u_{*}}{\omega}\right)}\left(\frac{0.01 K^{2} U^{3}}{g H \omega}-\frac{3 U J}{\omega}\right) \frac{d C}{d y}\right]=0
\end{aligned}
$$

where $u_{*}$ is friction velocity; $g$ is acceleration of gravity; $\mathrm{U}$ is vertical mean velocity of mixture $\int_{0}^{H} u_{m} d y=H U$. Equation (7) and (8) is function of density and flow velocity distribution. Because they are nonlinear differential equation, it is difficult to solution by analytical solution, and must use numerical analysis method with computer.

\section{MODEL OF NUMERICAL ANALYSIS}

\section{1 Building simulation model}

In our research, it uses cylindrical stirred tank and double helix blade (see Figure 1).The main parameters of cylindrical stirred tank are as follows: the total length is $1000 \mathrm{~mm}$, the 
total height is $525 \mathrm{~mm}$, the bottom radius of circular tank is $250 \mathrm{~mm}$, and the fluid height is $305 \mathrm{~mm}$. The main parameters of double helix blade are: the diameter of blade is $400 \mathrm{~mm}$, the helix pitch is $600 \mathrm{~mm}$, the number of blade is 3 pieces, and the distribution angle of helix blade is 180 degrees.

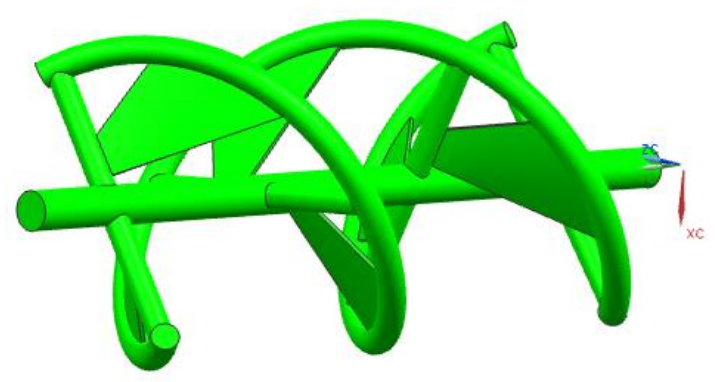

Figure 1. Stirred structure of double helix

Numerical calculation uses the Fluent platform [17]. In the process of numerical calculation, fried pepper sauce is simplified to solid-liquid two-phase flow. The density of rapeseed oil of liquid phase is $930 \mathrm{~kg} / \mathrm{m}^{3}$, and its viscosity is $0.4 \mathrm{p}$. The density of pepper particle of solid phase is $1280930 \mathrm{~kg} / \mathrm{m}^{3}$, its average diameter is $10 \mathrm{~mm}$, and the volume number is $75 \%$ [18-20].

The whole area is divided into liquid zone and solid zone. The liquid zone is calculated by hexahedral grid, and the solid zone uses tetrahedron grid $[21,22]$. In order to ensure the accuracy of calculation process, the grid is refined in some important areas such as interface of liquid-solid (see Figure 2), and place near wall for fluid, so the total number of grid is 214328 .

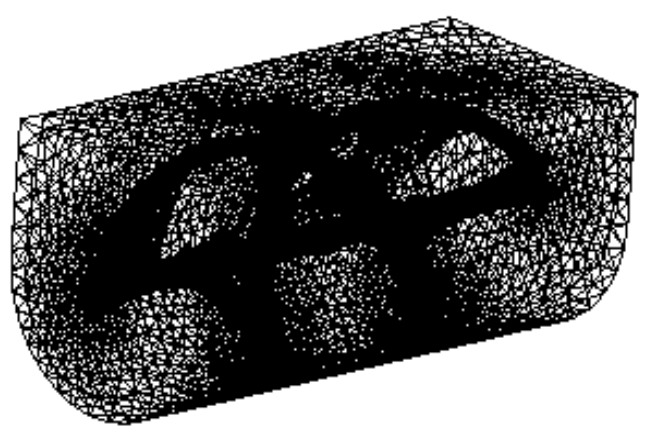

Figure 2. Grid model in computer

\section{2 Boundary condition}

The import is set velocity conditions to steady state, homogeneous, certain speed along the axial. The export is set flow conditions to free. The wall condition is set to no slip. A standard wall function is used to the near wall area. The interface between liquid and solid zone is set to rotate wall, which its speed is $5 \mathrm{rad} / \mathrm{s}$.

\section{RESULT ANALYSIS}

\section{1 Distribution of velocity field}

1. Velocity field of shaft section

To study the velocity distribution of solid-liquid two-phase flow, different shaft sections of velocity field are adopted to analyze comparatively, which are $X=0 \mathrm{~mm}, X=50 \mathrm{~mm}, X=-50$ $\mathrm{mm}, X=100 \mathrm{~mm}, X=-100 \mathrm{~mm}$. Figure 3 shows velocity distribution of five sections in stirred tank.

In the figure 3 , there are obvious intersection areas of radial and axial flow around blade in the stirred structure of double helix blade. There is main radial flow away from the blade area. There is obvious regional turbulence between blades under the action of helix blade, which promotes fluid mix degree between upper and lower layer. There is very poor liquidity near wall area in stirred tank because its speed is low, which cases a stir dead angle. Because flow field of blade is main radial flow and there is smaller axial flow near blade area, a large range of mixed flow is not formed, which causes that liquidity is poor. In addition, the different intensity turbulence area is formed in the center area of blade around fluid field. With the stirring of fried pepper sauce, the turbulence area is moving with helix style to promote fluid mix in different area of stirred tank. The top liquid is free surface, and it is easy to realize flow, so the average speed of fluid on the top of tank is large than the bottom of tank. In the bottom of tank, there are some influences of gravity and wall, so the liquidity of fluid is inhibited to some extent.

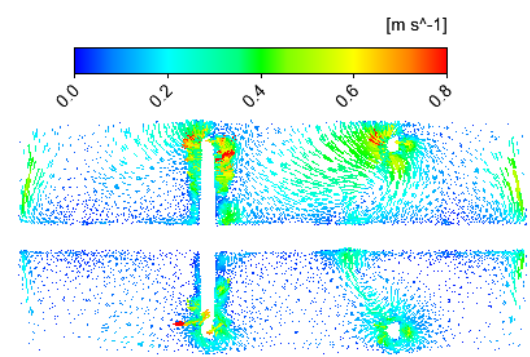

(a) $X=0 \mathrm{~mm}$

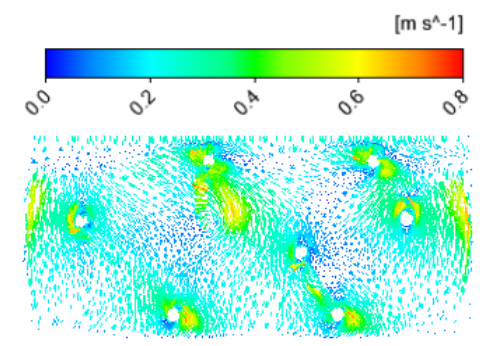

(b) $\mathrm{X}=50 \mathrm{~mm}$

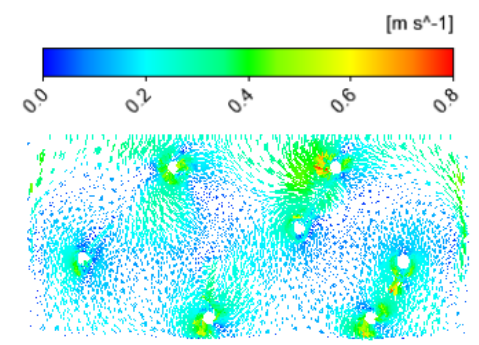

(c) $\mathrm{X}=-50 \mathrm{~mm}$

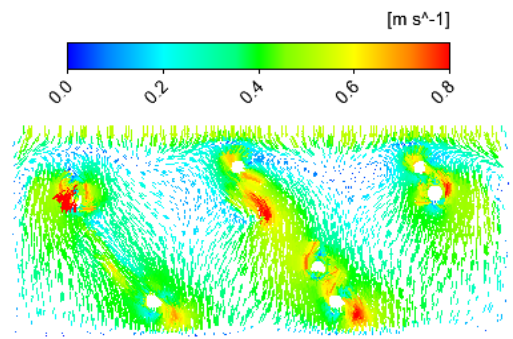

(d) $X=100 \mathrm{~mm}$ 


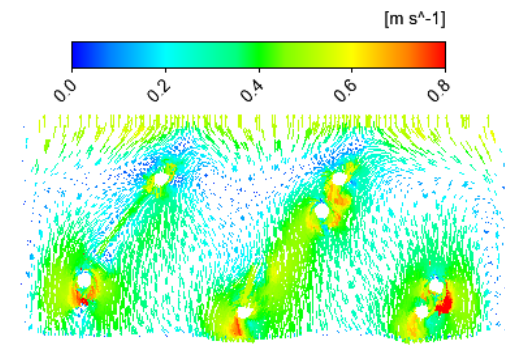

(e) $X=-100 \mathrm{~mm}$

Figure 3. The velocity vector diagram of different shaft section $(\mathrm{X})$ for the structure of double helix blade

2. Velocity field of longitudinal section

Two groups of different longitudinal section is selected to analyze velocity field: $Y=100 \mathrm{~mm}, Y=400 \mathrm{~mm}$ in the near wall area; $Y=200 \mathrm{~mm}, \mathrm{Y}=300 \mathrm{~mm}$ in the blade area (see Figure 4).

According to analysis result of figure 4 , it is conclude that, in the structure of double helix blade, the flow velocity of upper region is large and it is easy to form flow loop area; the flow velocity of bottom region is obviously lower than the upper region, so turbulent area almost does not exist. In short, the speed of upper region is large, and the speed of bottom region has certain increase, but the overall distribution is uniform. For the stirring shaft area, fluid speed is low, but the speed is gradually increased with increase of the radial distance, which form mixed flow with certain intensity. For the near wall area, according to the distribution of fluid velocity in figure 4 , the average speed of bottom region is larger. For the liquidity around the stirring shaft, it is best in the near axial area, because the propelling helix blade provides large axial flow speed to effectively promote uniform mixing of the different regions. In addition, from figure 4 , it is also show that the flow velocity and turbulence intensity of blade region is obviously larger than the near wall area, so fluid field in the blade region has large speed and obvious turbulence phenomenon, which promotes flow of upper and bottom region.

In a word, for the fluid in the stirred tank of double helix blade, the average flow velocity is large, and it is easy to form turbulence that has strong intensity, wide range, and good liquidity, but the flow characteristics is obvious decline in the near wall area.

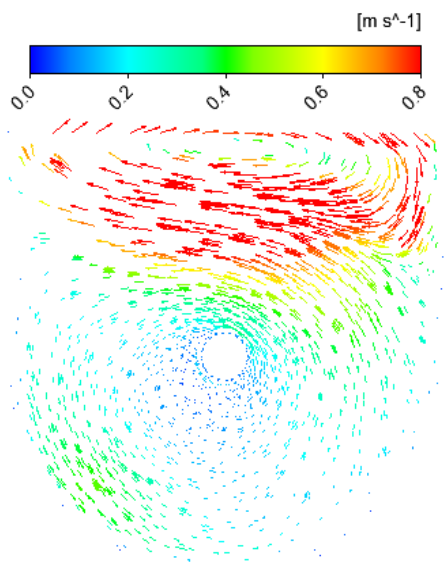

(a) $\mathrm{Y}=100 \mathrm{~mm}$

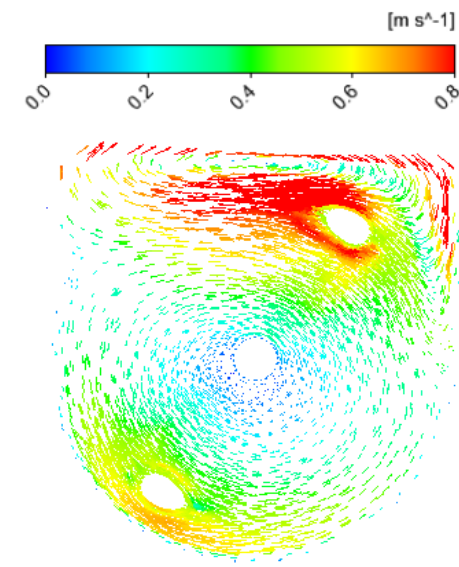

(b) $\mathrm{Y}=200 \mathrm{~mm}$

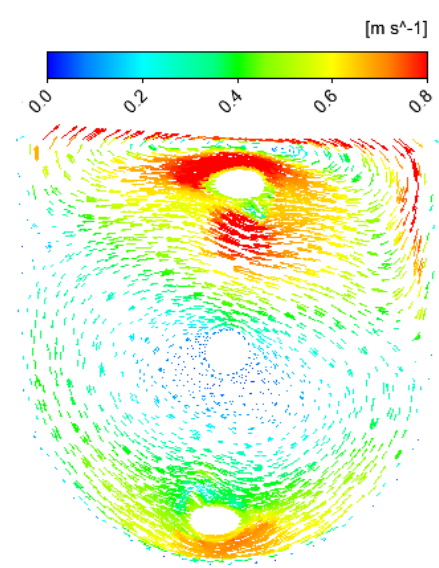

(c) $\mathrm{Y}=300 \mathrm{~mm}$

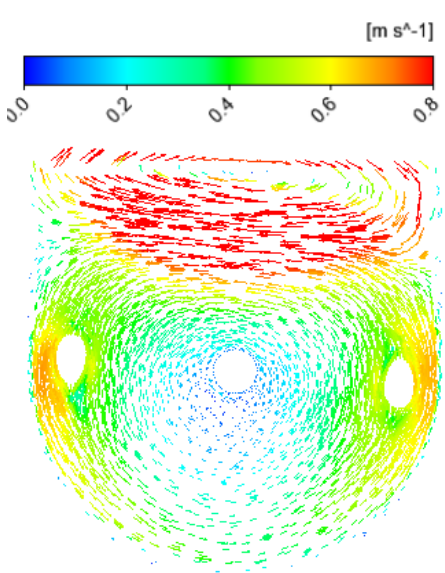

(d) $\mathrm{Y}=400 \mathrm{~mm}$

Figure 4. The velocity vector diagram of different longitudinal section (Y) for the structure of double helix blade

\section{2 Distribution of density field}

In the stirring of fried pepper sauce, density distribution of solid-liquid two-phase reflects uniformity degree of stirring, which is very important to fluid characteristics. Density distribution of solid-liquid two-phase is researched at $\mathrm{X}=0$ $\mathrm{mm}$ and $\mathrm{X}=100 \mathrm{~mm}$ of shaft section, $\mathrm{Y}=50 \mathrm{~mm}$ of longitudinal section in the near wall area, and $Y=250 \mathrm{~mm}$ of longitudinal section in the blade area (see Figure 5).

Figure 5 (a) and (b) show distribution of density field at 
$\mathrm{X}=0 \mathrm{~mm}$ and $\mathrm{X}=100 \mathrm{~mm}$ of shaft section. From the figures, density field in stirred tank has high density area and low density area to some extent, but entire distribution of density field is more uniform. Low density area at the top is small, and high density area at corner of the bottom is large.

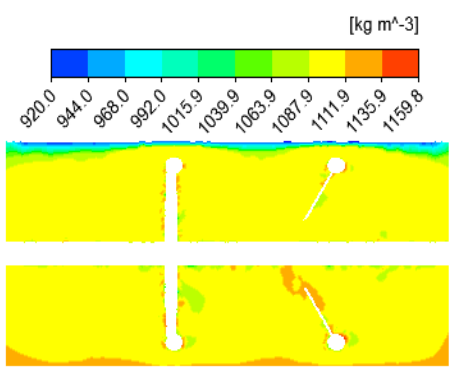

(a) $\mathrm{X}=0 \mathrm{~mm}$

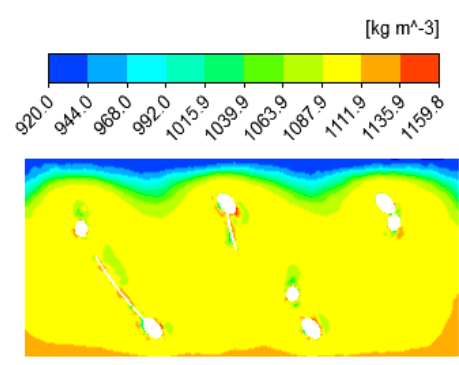

(b) $\mathrm{X}=100 \mathrm{~mm}$

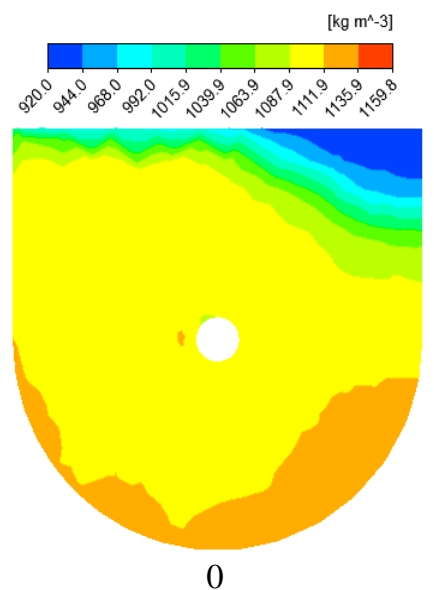

(c) $\mathrm{Y}=50 \mathrm{~mm}$

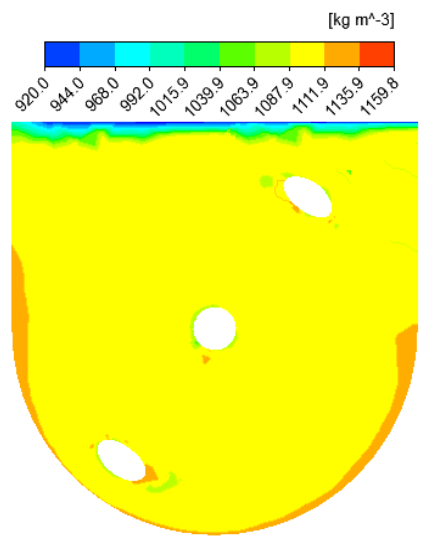

(d) $Y=250 \mathrm{~mm}$

Figure 5. The density distribution diagram of different shaft section (X) and longitudinal section (Y) for the structure of double helix blade
Figure 5 (c) shows density distribution in the near wall area It can be concluded that, in the mixing of solid-liquid twophase in stirred area, density distribution has uniform area to some extent. Density distribution is uniform in middle area. Low density area of the rapeseed oil is at the top. Accumulation area of high density is more at the bottom, which is likely to form a serious stirring dead corner.

Figure 5 (d) is density distribution in the stirring area. It is obtained by analysis that the stirring uniformity in the stirring area is more improvement than the near wall area, there are not obvious high density area and low density area in the density field of blade area, and the whole flow field tends to the state of uniform mixing. Thus, for the structure of double helix blade, the stirring uniformity in the middle of flow field is very good, and can realize a wide range of homogenization, but there is the stirring dead corner at the near wall area.

In a word, from the figure 5, the uniformity of density distribution is very good in the middle of stirring area, high and low density area is very less, and mixing performance is the best.

\section{CONCLUSION}

In this paper, the movement of solid-liquid two-phase flow is analyzed based on extreme theory of energy consumption. Velocity field and density field distribution of solid-liquid two-phase of fried pepper sauce in stirred tank of double helix blade is obtain by the Fluent platform. To analyze the computation result, there is following conclusion:

(1) From the above distribution diagram of velocity field, with the stirring of helix blade, flow speed at the upper region is large, and it is easy to form circulation area. Flow speed at the bottom region is lower than the upper, and the upper region almost has not turbulent area. In addition, flow speed and turbulent intensity in the blade area is obvious higher than the near wall area, and liquidity around the stirring shaft is also large.

(2) In the middle of stirring area, uniformity of density distribution in stirred tank of double helix blade is good, distribution of aggregated area of high and low density is very less, and mixing performance is the best.

In short, the paper obtains the flow velocity and density distribution of different section based on building flow model of mixture of fried pepper sauce in stirred tank, which provides data basis for automatic filling technology. Because the movement of solid-liquid two-phase is very complexity, the research in the paper is performed under the condition of certain basic assumptions. In the future, experimental method is used to measure, because it is not only to provide boundary conditions for numerical calculation, but also further verify the result of computational results.

\section{ACKNOWLEDGEMENTS}

This research is supported by Guizhou Province Science and Technology Fund Project (Guizhou Branch Total LH [2014] No. 7645, 7644).Guizhou Province Science and Technology Fund Project (Guizhou Branch Total JZ [2014] No. 2004; Guizhou Branch Total [2015] No. 13). 


\section{REFERENCES}

[1] Liu Yan-Min, Wu Yong-Jun, Wang Ya-Juan, et al., "Volatile Flavor Components Analysis of Fried Pepper Sauce in Guizhou Province," Food Science, vol. 34, no. 20, pp. 115-120, 2013. DOI: $10.7506 / \mathrm{spkx} 1002-$ 6630-201320047.

[2] Dohi N., Takahashi T., Minekawa K., et a1. "Power consumption and solid suspension performance of large-scale impellers in gas-liquid-solid three-phase stirred tank reactors, " Chemical Engineering Journal, vol. 97, no. 2-3, pp. 103-114, 2009. DOI: 10.1016/S1385-8947(03)00148-7.

[3] Ochieng A., Onyango M. S., "CFD simulation of solids suspension in stirred tanks: Review," Hem Ind, vol. 64, no. 5, pp. 365-374, 2010. DOI: 10.2298/HEMIND1007140510.

[4] Montante G. and Magelli F., "Modelling of solids distribution in stirredtanks: Analysis of simulation strategies and comparison with experimental data," International Journal of Computational Fluid Dynamics, vol. 19, no. 3, pp. 253-262, 2005. DOI: $\underline{10.1080 / 10618560500081795 .}$.

[5] Giuseppina M., Alessandro P. and Franco M., "Analysis of dilute solid-liquid suspensions in turbulent stirred tanks," Chemical Engineering Research and Design, no. 1, pp. 1-9, 2012. DOI: 10.1016/i.cherd.2012.01.009.

[6] E. Dotsika, D. Poutoukis and B. Raeo. "Stable isotope composition of Hellenic bottled Water". Journal of Geochemical Exploration, vol. 107, pp. 299-304, 2010. DOI: 10.1016/j.gexplo.2010.07.002.

[7] Guanghui Liu, Weidong Cao, Yue Li, et al., "Study on solid-liquid two-phase unsteady flow in multistage pump," Fluid Machinery and Fluid Engineering, 2014 ISFMFE - 6th International Symposium on, 2014, pp. 1-8. DOI: $10.1049 / \mathrm{cp} .2014 .1244$

[8] Huang Zhonghua, Cao Yue and Xie Ya, "Polymetallic Sulfide Particle Hydraulic Lifting Performance Simulation," Intelligent Computation Technology and Automation (ICICTA), pp. 423 - 427, 2014. DOI: 10.1109/ICICTA.2014.108.

[9] Alexander Ledkov and Songjing Li, "Flow forces acting on particles in the flow in a well and slotted filter," Fluid Power and Mechatronics (FPM), pp. 283 - 288, 2015. DOI: 10.1109/FPM.2015.7337126.

[10] Hee Cheen No.. “On Soo's equations in multidomain multiphase fluid mechanics". Int. J. Multiphase Flow, vol. 8, pp. 297-299, 1982. DOI: 10.1016/03019322(82)90039-8.

[11] Kaempfer Th U. and Rappaz M., "Welding and
Advanced Solidification Processes," In: Sahm P. R., ed., Proc 9th CoIV on Modeling of Casting, Aachen, TMS, 2000, pp. 640. DOI: 10.1049/cp.2014.1156.

[12] Chen Zhao, Huang Yong, Jiang Jun and Wang Jun. "Heat Transfer Characteristics of a Solid-Liquid Mixture Rotation Baffle Heat Exchanger," 2012 AsiaPacific Power and Energy Engineering Conference, 2012, pp. 1-6. DOI: 10.1109/APPEEC.2012.6307040.

[13] Alexander Ledkov and Songjing Li, "Flow forces acting on particles in the flow in a well and slotted filter." Fluid Power and Mechatronics (FPM), pp. 283 - 288, 2014. DOI: 10.1109/FPM.2015.7337126.

[14] Iraj Zandi, Advances in Solid-Liquid Flow in Pipes and Its Application, Pergamon Press Inc., 2013. DOI: 10.1109/ICMA.2009.5246584.

[15] Pascal Clausen, Martin Wicke, et al., "Simulating liquids and solid-liquid interactions with Lagrangian meshes." ACM Transactions on Graphics, 2014, pp. 121-132. DOI: 10.1145/2451236.2451243.

[16] R. K. Sharma, P. Ganesan, et al., "Developments in organic solid-liquid phase change materials and their applications in thermal energy storage," Energy Conversion and Management, vol. 3, no. 95, pp. 193228, 2015. DOI: 10.1016/j.enconman.2015.01.084.

[17] Fluent Inc., FLUENT User's Guide, Fluent Inc., 2003.

[18] Yuan Shouqi, Zhang Peifang, Zhang Jinfeng, et a1., "Numerical simulation of 3-d dense solid--liquid twophase turbulent flow in a nonclogging mud pump," Chinese Journal of Mechanical Engineering, vol. 17, no. 4, pp. 623-627, 2004.

[19] Rudolf S., "Numerical simulation of the two-phase flow in centrifugal pump impellers," ASME Journal Fluids Engineering, vol. 123, no. 1, pp. 1-6, 2001. DOI: $10.1115 /$ FEDSM2002-31193.

[20] Shum Y. K. E., Tan C. S. and Cumpsty N. A., "Impeller-diffuser interaction in a centrifugal compressor," Journal of Turbomachinery, vol. 122, no. 4, pp.777-786, 2000. DOI: $\underline{10.1115 / 2000-G T-}$ 0428.

[21] Zhao Binjuan, Yuan Shouqi, Liu Houlin, et a1., "Three-dimensional coupled impeller-volute simulation of flow in a centrifugal pump and performance prediction," Chinese Journal of Mechanical Engineering, vol. 19, no. 1, pp. 59-62, 2006.

[22] Wei Han, Ting Yue, Rennian Li, et al., The numerical study of transient flow in rotor and stator cascades of solid-liquid two-phase flow," Fluid Machinery and Fluid Engineering, pp. 1-5, 2014. DOI: 10.1049/cp.2014.1156. 